\title{
Avaliação do Serviço de Ônibus Interno no Campus Sede da UFSM
}

Internal Bus Service Evaluation at UFSM Campus

\begin{abstract}
Siomara Cristina Broch', Nicásio Gouveia", Guilherme Steavie"II, Felipe Sehnem Mattana'v, Fernando de Jesus Moreira Juniorv
\end{abstract}

\begin{abstract}
RESUMO
Este artigo apresenta os resultados de uma pesquisa de opinião sobre o serviço de transporte interno de ônibus do campus sede da UFSM, realizada em 2015. A pesquisa mostrou os principais pontos de satisfação e insatisfação em relação ao funcionamento da linha na época. Os dados foram coletados através de formulário eletrônico do Google Docs, acessado via e-mail, resultando numa amostra de 915 estudantes matriculados nos diversos cursos de graduação e pós-graduação do campus (5,3\% da população). Os principais resultados mostraram que $20 \%$ dos respondentes não conhecem este serviço e que o predomínio de uso do serviço é o diurno. Os usuários mostraram maior insatisfação no item climatização interna do veículo e no estado de conservação. Os itens horário, frequência e rota de circulação do ônibus, quantidade de lugares e pontualidade tiveram alto índice de satisfação. Conclui-se que é necessária maior divulgação do serviço, horários e rotas de circulação, especialmente para os estudantes de cursos noturnos, e melhoria nas condições físicas do veículo.
\end{abstract}

Palavras-chave: Pesquisa de satisfação; Análise exploratória; Qualidade de serviço.

\begin{abstract}
This article presents the results of a survey about the internal bus service from UFSM, held in 2015. The survey showed the main points of satisfaction and discontent regarding the operation of the line at the time. Data were collected through an electronic Google Docs form, accessed via email, resulting in a sample of 915 students enrolled in the various undergraduate and graduate campus courses (5.3\% of the population). The main results showed that $20 \%$ of respondents do not know this service and the predominance of use of the service is the daytime. Users showed greater dissatisfaction with the vehicle's internal climate control and bus condition. The items time, frequency and route of the bus, number of seats and punctuality had a high level of satisfaction. It is concluded that greater disclosure of the service and its characteristics is necessary, especially for students of night courses, and improvement in the physical condition of the vehicle.
\end{abstract}

Keywords: Satisfaction survey; Exploratory analysis; Service quality.

\footnotetext{
'Universidade Federal de Santa Maria, Santa Maria, Brasil. E-mail: siomara.lago@iffarroupilha.edu.br.

" Universidade Federal de Santa Maria, Santa Maria, Brasil. E-mail: nicasiogouveia@yahoo.com.br.

III Universidade Federal de Santa Maria, Santa Maria, Brasil. E-mail: gs.staevie@gmail.com.

IV Universidade Federal de Santa Maria, Santa Maria, Brasil. E-mail: felipe.mattana@gmail.com.

v Universidade Federal de Santa Maria, Santa Maria, Brasil. E-mail: fmjunior777@yahoo.com.br.
} 


\section{INTRODUÇÃO}

Em estudos da geografia urbana, o deslocamento nas cidades é analisado e interpretado em termos de um esquema conceitual que articula a mobilidade urbana, que são as massas populacionais e seus movimentos; a rede, representada pela infraestrutura que canaliza os deslocamentos no espaço e no tempo; e os fluxos, que são as macro decisões ou condicionantes que orientam o processo no espaço (RAIA JUNIOR, 2000). Então, os princípios e as estratégias de planejamento e gestão da mobilidade são aplicáveis em qualquer área geográfica, seja um lugar específico ou uma cidade.

De acordo com Castro (2005), este conceito abrange ações numa ampla escala espacial e pode-se implantar em países com diferente localização geográfica e plano nacional de mobilidade, o qual demonstra a sua flexibilidade e aplicabilidade, desde que se considerem as especificidades locais. Desta forma, também é aplicável aos diversos Polos Geradores de Viagens - PGV como shoppings, prédios de escritórios, hospitais, Universidades, dentre outros. Em uma cidade, um Campus Universitário se torna um ponto a destacar, ao converter-se num PGV. Tanto dentro, quanto fora do Campus, os problemas da mobilidade são representativos (PARRA, 2006). Portanto, a gestão da mobilidade torna-se uma atividade importante para orientar o processo de desenvolvimento do espaço e nos investimentos na circulação.

Tobias (2013) destaca que a mobilidade está relacionada aos deslocamentos diários de pessoas no espaço urbano, o que leva em consideração não apenas sua efetiva ocorrência, mas também a sua possibilidade de ocorrência. O transporte coletivo é um meio de mobilidade interna dentro do campus universitário, podendo ser enquadrado como um serviço, o que o diferencia das demais atividades no que diz respeito a qualidade. Lima Junior (1995) destaca como pontos de diferenciação entre transporte e as demais atividades: a intangibilidade de seus resultados; a participação do cliente na produção; a heterogeneidade dos processos e resultados, com alta variabilidade motivada por fatores de difícil controle, principalmente os associados ao cliente; os picos de demanda e o ambiente não controlado de produção, o qual se desloca espacialmente. 
A definição de qualidade pode variar. De acordo com Juran (1990), a qualidade é vista como a adequação ao uso. Um produto com qualidade apresenta características que atendam às necessidades e aumentam a satisfação do cliente. Entretanto, a qualidade é definida por Deming (1990) como sendo o nível de satisfação dos clientes, propondo que a qualidade é construída e não apenas inspecionada. A satisfação do cliente pode ser abordada como uma avaliação a ser realizada pelo próprio cliente no período pós-consumo, que considera se a alternativa escolhida atende ou não às expectativas que ele tinha quando procurou o produto ou serviço adquirido (ENGEL et al., 2000).

Segundo Paulley (2006), a qualidade do serviço de transporte pode ser definida por uma ampla gama de atributos nos quais podem ser influenciados por autoridades responsáveis pelo planejamento e operadores de transporte. Os principais atributos para a qualidade do transporte público são: o tempo de acesso ao ponto de embarque e hora de saída do ponto de desembarque, intervalo de serviço, tempo gasto dentro do veículo, o ambiente de espera, as características do veículo e o fornecimento de informações sobre o serviço que está sendo prestado. Dentro desse contexto, desde agosto de 2014 os estudantes, técnico-administrativos em educação e os docentes da Universidade Federal de Santa Maria (UFSM) contam com o transporte interno gratuito, nomeado de "Via UFSM", no Campus sede - de Camobi). O veículo, com 22 lugares, faz um percurso interno, atendendo a um itinerário que contempla grande parte do espaço territorial da UFSM. O serviço funciona a cada meia hora, das $7 \mathrm{~h} 30 \mathrm{~min}$ às $22 \mathrm{~h}$, de segunda à sexta-feira, exceto feriados, sempre com saída do Centro Comercial do Campus localizado no posto de combustível após o arco e pode ser utilizado mediante comprovação de vínculo com a UFSM.

Devida a importância do serviço de ônibus interno na universidade, dado que o campus sede possui uma ampla extensão territorial, neste sentido, realizou-se um estudo com o objetivo de obter informações sobre o serviço de ônibus interno da Universidade Federal de Santa Maria prestado aos acadêmicos matriculados nos cursos de graduação e pós-graduação desta instituição em 2015. Este estudo foi realizado como uma atividade de ensino da disciplina de Pesquisa de Opinião e de 
Mercado do Curso de Bacharelado em Estatística. A pesquisa foi aprovada no Comitê de Ética em Pesquisa (CEP) da UFSM com o Certificado de Apresentação para Apreciação Ética (CAAE) n 49645915.8.0000.5346 por meio do parecer $n^{\circ} 1.290 .388$ de 21 de outubro de 2015.

\section{METODOLOGIA}

Definiu-se como população alvo os alunos que frequentam o campus sede da UFSM, que compreende aproximadamente 18.077 acadêmicos com base no número total de matriculados. Segundo McDaniel e Gates (2005), os índices de resposta das pesquisas aplicadas por meio da internet variam entre 30\% e 60\%. Dessa forma se esperava que amostra fosse composta entre 5.424 e 10.847 alunos com dos diversos cursos de graduação e pós-graduação.

Considerou-se como critério de inclusão todos os acadêmicos matriculados em cursos situados no campus sede que concordam em participar voluntariamente da pesquisa, sendo ou não usuários do sistema de transporte interno da UFSM. A população alvo foi contatada e convidada a participar da pesquisa por e-mail. Os emails dos alunos, que estão cadastrados no SIE - Sistema de Informações para o Ensino da UFSM, foram solicitados junto ao CPD - Centro de Processamento de Dados da UFSM. No e-mail constava o link para a participação na pesquisa. Aqueles alunos que acessaram o link e concordaram em participar da pesquisa responderam um questionário on-line auto-aplicável, de fácil entendimento e utilização, elaborado no Google Docs (www.google.com/docs), pacote de aplicativos do Google.

No estudo foi utilizada uma amostra não-probabilística por conveniência, pois os pesquisadores se utilizaram dos indivíduos mais disponíveis da população, que atenderam ao convite da pesquisa, que é o caso das pesquisas pela internet. Neste caso, o indivíduo não foi selecionado aleatoriamente, mas ele decidiu se queria participar ou não da pesquisa. Algumas vantagens da utilização da internet na aplicação do questionário são: maior alcance, economia de tempo, baixo custo, facilidade de coleta e tabulação dos dados, grande retorno de respostas. A maior desvantagem é que não se trata de uma amostra probabilística, não sendo, portanto, possível fazer inferências, já que não são estabelecidos o nível de confiança e o erro 
amostral. Isso pode, de certa forma, comprometer a confiabilidade da pesquisa se comparada com uma amostragem aleatória. No entanto, as vantagens de uma pesquisa on-line superam em muito as desvantagens de uma pesquisa tradicional, segundo McDaniel e Gates (2005), considerando que o comprometimento da confiabilidade da amostra pode ser minimizado pelo fato de que a amostra poderá ser muito maior do que seria numa pesquisa tradicional limitada pelos custos e pelo tempo. A análise dos dados foi realizada por meio de estatística descritiva, em que inicialmente os dados foram organizados em gráficos e tabelas. Posteriormente foi elaborado um relatório e encaminhado para a Pró-Reitoria de Infraestrutura (Proinfra) da UFSM explicitando todas as conclusões e sugestões de melhorias, evidenciadas através dos resultados obtidos.

\section{RESULTADOS E DISCUSSÕES}

Apesar da literatura indicar um índice de respostas de 30\% a 60\% para pesquisas com esta forma de coleta de dados (MCDANIEL; GATES, 2005), a amostra deste estudo totalizou 915 respostas, ou seja 5,3\% da população alvo. A restrição de tempo de aplicação, que ficou aberto durante apenas 30 dias, e o fato de que muitos dos estudantes que receberam o e-mail para a pesquisa não utilizam o serviço, podem ter sido fatores influentes para uma amostra menor. Porém, trata-se de uma amostra considerável para o estudo, mesmo sendo não-probabilística, já que é um estudo piloto, incipiente, tendo foco descritivo de levantamento de informações. Pelos dados apresentados na Tabela 1, 20\% dos respondentes à pesquisa não conhecem o serviço de ônibus interno da UFSM, ou seja, 1 a cada 5 respondentes da pesquisa não conhece o serviço, o que pode ser considerado um alto percentual já que o serviço é estendido gratuitamente a todos.

Tabela 1 - Frequência de respondentes que conhecem o serviço de ônibus interno da UFSM

\begin{tabular}{lcc}
\hline Conhecem o serviço & Frequência & Porcentagem \\
\hline Não & 179 & $20 \%$ \\
Sim & 736 & $80 \%$ \\
\hline Total & 915 & $100 \%$ \\
\hline
\end{tabular}


Dentre os pesquisados que conhecem o serviço de ônibus interno da UFSM, 64\% fazem uso dele, conforme a Tabela 2, o que é um valor expressivo.

Tabela 2 - Frequência de uso do serviço de ônibus interno da UFSM

\begin{tabular}{lll}
\hline Usa o serviço & Frequência & Porcentagem \\
\hline Não & 265 & $36 \%$ \\
Sim & 471 & $64 \%$ \\
\hline Total & 736 & $100 \%$ \\
\hline
\end{tabular}

As análises a seguir foram realizadas sobre os 471 respondentes que utilizam o serviço. Sobre o principal motivo do uso do serviço de ônibus interno, os pesquisados relatam que utilizam para economizar tempo e que há a necessidade de deslocamentos devido a disciplinas, estágio, trabalho, experimentos em diferentes e distantes locais, conforme as informações da Tabela 3.

Tabela 3 - Principal motivo da utilização do serviço de ônibus interno da UFSM

\begin{tabular}{lcc}
\hline Principal motivo para a utilização do serviço & Frequência & Porcentagem \\
\hline Economia de tempo & 138 & $29 \%$ \\
Faço cursos e/ou disciplinas em locais diferentes & 127 & $27 \%$ \\
O deslocamento a pé é cansativo & 100 & $21 \%$ \\
Tenho bolsa de estudos em um prédio distante do prédio do meu curso & 63 & $13 \%$ \\
Deslocamento interno longo rotineiramente & 23 & $5 \%$ \\
Deslocamento em dias de chuva & 5 & $1 \%$ \\
Preciso ir onde somente o ônibus interno passa & 3 & $1 \%$ \\
Moro nos arredores da UFSM e o transporte interno me auxilia & 3 & $1 \%$ \\
Outros & 5 & $1 \%$ \\
Não respondeu & 4 & $1 \%$ \\
\hline Total & 471 & $100 \%$ \\
\hline
\end{tabular}

Quanto à idade, 94\% dos usuários do serviço de ônibus interno da UFSM têm entre 18 e 35 anos, com predomínio de 18 a 25 anos (70\%), conforme dados da Tabela 4. As informações da Tabela 5 mostram que o turno de maior uso do serviço é o diurno, pela manhã e à tarde, com $69 \%$ dos usuários fazendo uso deste serviço nestes horários. Os dados da Tabela 6 são obtidos a partir da Tabela 5, em que o número de usuários é quantificado, considerando que uma pessoa utiliza o serviço mais de um turno.

Já na frequência de uso do serviço, conforme a Tabela 7 predomina o uso eventual (43\%), seguido do uso diário (22\%) e o uso em um ou dois dias da semana (21\%). 
Tabela 4 - Idade dos respondentes que utilizam o serviço de ônibus interno da UFSM

\begin{tabular}{lcc}
\hline Idade & Frequência & Porcentagem \\
\hline Menos de 18 anos & 7 & $1,5 \%$ \\
Entre 18 à 25 anos & 331 & $70,3 \%$ \\
Entre 26 à 35 anos & 111 & $23,6 \%$ \\
Entre 36 à 45 anos & 13 & $2,8 \%$ \\
46 anos ou mais & 8 & $1,7 \%$ \\
Não respondeu & 1 & $0,2 \%$ \\
\hline Total & 471 & $100,0 \%$ \\
\hline
\end{tabular}

Tabela 5 - Turno(s) em que os respondentes que utilizam o serviço de ônibus interno frequentam a UFSM

\begin{tabular}{lcc}
\hline Turno & Frequência & Porcentagem \\
\hline Manhã/Tarde & 323 & $68,6 \%$ \\
Manhã/Tarde/Noite & 90 & $19,1 \%$ \\
Somente Manhã & 22 & $4,7 \%$ \\
Somente Tarde & 20 & $4,2 \%$ \\
Somente Noite & 6 & $1,3 \%$ \\
Manhã/Noite & 5 & $1,1 \%$ \\
Tarde/Noite & 4 & $0,8 \%$ \\
Não respondeu & 1 & $0,2 \%$ \\
\hline Total & 471 & $100,0 \%$ \\
\hline
\end{tabular}

Tabela 6 - Frequência de uso do serviço de ônibus interno na UFSM, por turno

\begin{tabular}{lcc}
\hline Turno & Frequência & Porcentagem \\
\hline Manhã & 440 & $93,4 \%$ \\
Tarde & 437 & $92,8 \%$ \\
Noite & 105 & $22,2 \%$ \\
\hline
\end{tabular}

Tabela 7 - Frequência de uso do serviço de ônibus interno da UFSM

\begin{tabular}{lcc}
\hline Uso do serviço & Frequência & Porcentagem \\
\hline Diariamente & 103 & $21,9 \%$ \\
Um ou dois dias na semana & 100 & $21,2 \%$ \\
Três ou quatro dias na semana & 64 & $13,6 \%$ \\
Eventualmente & 203 & $43,1 \%$ \\
Não respondeu & 1 & $0,2 \%$ \\
\hline Total & 471 & $100,0 \%$ \\
\hline
\end{tabular}

A Tabela 8 apresenta os níveis de satisfação dos usuários que utilizam o serviço de ônibus interno da UFSM para sete características avaliadas na pesquisa. Ao todo, 470 repostas foram obtidas, pois um pesquisado não respondeu a nenhuma destas questões. 
Tabela 8 - Frequência absoluta (f) e percentual (\%) do nível de satisfação dos respondentes que utilizam o serviço de ônibus interno da UFSM

\begin{tabular}{lcccccccccc}
\hline Item avaliado & \multicolumn{2}{c}{$\begin{array}{c}\text { Muito } \\
\text { Satisfeito }\end{array}$} & \multicolumn{2}{c}{ Satisfeito } & \multicolumn{2}{c}{ Indiferente } & \multicolumn{2}{c}{ Insatisfeito } & \multicolumn{2}{c}{$\begin{array}{c}\text { Muito } \\
\text { Insatisfeito }\end{array}$} \\
\cline { 2 - 12 } & $\mathrm{f}$ & $\%$ & $\mathrm{f}$ & $\%$ & $\mathrm{f}$ & $\%$ & $\mathrm{f}$ & $\%$ & $\mathrm{f}$ & $\%$ \\
\hline $\begin{array}{l}\text { Horário de circulação } \\
\text { do ônibus }\end{array}$ & 77 & $16,3 \%$ & 248 & $52,7 \%$ & 42 & $8,9 \%$ & 79 & $16,8 \%$ & 24 & $5,1 \%$ \\
$\begin{array}{l}\text { Frequência de circulação } \\
\text { dos ônibus }\end{array}$ & 116 & $24,6 \%$ & 206 & $43,7 \%$ & 28 & $5,9 \%$ & 89 & $18,9 \%$ & 31 & $6,6 \%$ \\
$\begin{array}{l}\text { Estado de conservação } \\
\text { dos ônibus }\end{array}$ & 40 & $8,5 \%$ & 136 & $28,9 \%$ & 134 & $28,5 \%$ & 110 & $23,4 \%$ & 50 & $10,6 \%$ \\
$\begin{array}{l}\text { Rota de circulação } \\
\text { do ônibus }\end{array}$ & 87 & $18,5 \%$ & 246 & $52,2 \%$ & 36 & $7,6 \%$ & 72 & $15,3 \%$ & 29 & $6,2 \%$ \\
$\begin{array}{l}\text { Quantidade de lugares } \\
\text { (assentos) no ônibus }\end{array}$ & 72 & $15,3 \%$ & 225 & $47,8 \%$ & 75 & $15,9 \%$ & 65 & $13,8 \%$ & 33 & $7,0 \%$ \\
$\begin{array}{l}\text { Climatização interna } \\
\text { Pontualidade }\end{array}$ & 33 & $7,0 \%$ & 135 & $28,7 \%$ & 129 & $27,4 \%$ & 130 & $27,6 \%$ & 43 & $9,1 \%$ \\
\hline
\end{tabular}

Quanto ao horário de circulação do ônibus, 69\% dos usuários estão satisfeitos e $22 \%$ estão insatisfeitos, sendo um bem avaliado que precisa ser mantido ou melhorado. Quanto à frequência de circulação dos ônibus, de meia em meia hora, 68\% dos usuários estão satisfeitos e $26 \%$ insatisfeitos, também um item bem avaliado que deve ser mantido ou melhorado. Quanto ao estado de conservação dos ônibus apenas $37 \%$ dos usuários estão satisfeitos enquanto que 34\% estão insatisfeitos e $29 \%$ indiferentes foi um item mal avaliado, o que necessita ser reavaliado pelos responsáveis pelo serviço. Quanto à rota de circulação do ônibus $71 \%$ dos usuários estão satisfeitos com os pontos de paradas do ônibus, enquanto que $22 \%$ estão insatisfeitos, sendo outro item bem avaliado que deve ser mantido ou melhorado. Quanto à quantidade de lugares (assentos) no ônibus, 63\% dos usuários estão satisfeitos considerando suficientes e/ou adequados ao trajeto e objetivo do serviço, enquanto que $21 \%$ mostram-se insatisfeitos, sendo, portanto, outro item bem avaliado. Quanto à pontualidade do ônibus, 71\% dos usuários estão satisfeitos e 17\% insatisfeitos, sendo outro item bem avaliado que deve ser mantido ou pode ser melhorado. Quanto à climatização interna do veículo, considerada a temperatura, ventilação e conforto térmico interno do transporte, apenas 36\% dos usuários dizemse satisfeitos enquanto $37 \%$ estão insatisfeitos e $27 \%$ indiferentes. Este item é considerado mal avaliado pelos pesquisados, e pode melhorar muito a satisfação percebida pelos usuários, principalmente os indiferentes. 
A Figura 1 faz um comparativo dos níveis de satisfação e insatisfação dos usuários do ônibus interno da UFSM nos diversos itens analisados

Figura 1 - Gráfico comparativo dos níveis de satisfação e insatisfação dos respondentes que utilizam o serviço de ônibus interno da UFSM nos diversos itens pesquisados, em porcentagens

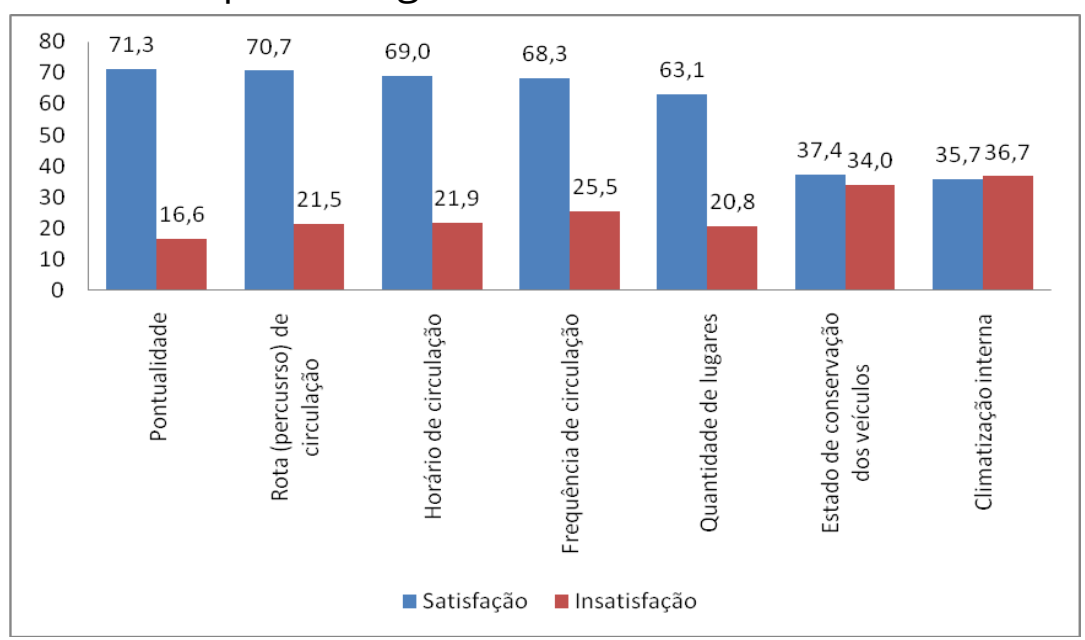

Observa-se que os itens pesquisados que os usuários mostraram maior satisfação foram a pontualidade do veículo e a rota de circulação, ambos com 71\% dos usuários satisfeitos. Já os itens de maior insatisfação foram a climatização interna do veículo, com 37\% de insatisfeitos, e o estado de conservação dos ônibus, com 34\% dos usuários insatisfeitos. Ao se analisar as sugestões, observou-se que 137 (29\%) indivíduos deram 208 sugestões com relação ao horário, à rota (percurso), ao serviço, ao veículo; geral, conforme mostra a Tabela 9.

Quanto ao horário de circulação do veículo verifica-se uma necessidade de ajuste. Os usuários fazem várias sugestões que, se analisadas em conjunto, se complementam. Inicialmente, é apontado um desajuste entre horários do ônibus e horários de aula. Na UFSM é usual início das aulas em hora exata ou meia hora, por exemplo, aulas iniciam 7h30min, 8h, 8h30min, e assim sucessivamente até as $21 \mathrm{~h}$. Portanto, a necessidade do serviço apontada pelos pesquisados é a partir das 7h da manhã. Portanto, a necessidade do serviço apontada pelos pesquisados é a partir das 7h da manhã. Além disso, argumentam que em alguns horários é necessária maior frequência de ônibus, talvez de 15 em 15 min, com o indicativo que existem horários com maior demanda, em que o ônibus fica cheio e até falta lugares. Assim, a 
implementação de ajustes de horários e estudo de horários com maior frequência é uma necessidade apontada de melhoria do serviço.

Outro aspecto de melhoria está relacionado com a rota do ônibus. A solicitação de passagem do veículo em alguns lugares ainda não atendidos e a explanação do desconhecimento da rota ou de mudanças na rota sem conhecimento evidencia a necessidade de ajustes. A sugestão de horários com rotas alternadas, incluindo alguns pontos em todos os turnos, bem como que o ônibus se desloque pela Avenida Roraima até a rótula, pois muitos estudantes residem nas proximidades do campus. Outra sugestão é a implantação de dois veículos em paralelo, sentidos opostos, atendendo uma maior demanda de ida e vinda dos usuários.

Quanto ao serviço em geral, uma sugestão com grande demanda é a divulgação do serviço. Neste aspecto surgem muitas sugestões interessantes: implantar aplicativo para celular com as informações atualizadas de horários e rotas; a organização interna da rota, com sinalização das paradas, identificação visual do ônibus; usar um sistema sonoro de informação interna no veículo sobre a rota que será seguida naquele horário. Este é, portanto, um ponto que, segundo os pesquisados, exige melhoria. Quanto ao veículo, é interessante observar a simpatia dos usuários com o ônibus utilizado. No entanto, apontam a necessidade de modernização e manutenção em todos os aspectos: mecânica, ergonômica, segurança e conforto, incluindo a necessidade de preparo dos motoristas para o serviço e a preocupação com a acessibilidade ao serviço. 
Tabela 9 - Sugestões levantadas na pesquisa para a melhoria do serviço de ônibus interno da UFSM

\begin{tabular}{|c|c|c|}
\hline Sugestão & \multirow{2}{*}{ Frequência } & \multirow{2}{*}{ Porcentagem } \\
\hline Sobre o horário & & \\
\hline Maior frequência nos horários de pico e menos em outros & 16 & $12 \%$ \\
\hline Frequência mínima de 30 minutos entre veículos (15 minutos) & 15 & $11 \%$ \\
\hline Rigidez no horário de saída (pontualidade) & 12 & $9 \%$ \\
\hline Iniciar a rota as 7 h da manhã & 11 & $8 \%$ \\
\hline Serviço a noite (rota especial) & 11 & $8 \%$ \\
\hline Mudança de horário (divergência em relação as aulas) & 7 & $5 \%$ \\
\hline Ônibus maior no horário do meio-dia & 6 & $4 \%$ \\
\hline Mais lugares no ônibus & 5 & $3 \%$ \\
\hline Subtotal & 83 & $61 \%$ \\
\hline \multicolumn{3}{|l|}{ Sobre a rota (percurso) } \\
\hline Manter na rota o Hospital Veterinário e Zootecnia & 9 & $7 \%$ \\
\hline Ônibus ir até a rótula & 7 & $5 \%$ \\
\hline Manter na rota a estrade de pinus & 5 & $3 \%$ \\
\hline Incluir as Ciências da saúde na rota & 4 & $3 \%$ \\
\hline Rota passar pela casa do estudante & 4 & $3 \%$ \\
\hline Mais de uma rota alternada & 4 & $3 \%$ \\
\hline Incluir o Tambo na rota & 4 & $3 \%$ \\
\hline Incluir na rota o Jardim Botânico & 3 & $2 \%$ \\
\hline Veículos paralelos com deslocamento em sentidos opostos & 3 & $2 \%$ \\
\hline Incluir a Fitotecnia na rota & 2 & $1 \%$ \\
\hline Incluir horário com rota até a estrada do Pains & 1 & $1 \%$ \\
\hline Subtotal & 46 & $34 \%$ \\
\hline \multicolumn{3}{|l|}{ Sobre o serviço } \\
\hline Divulgação dos horários, rota e pontos de parada & 19 & $14 \%$ \\
\hline Informar no ônibus a rota do dia (mudanças de rota) & 11 & $8 \%$ \\
\hline Sinalização exclusiva para as paradas do ônibus interno & 7 & $5 \%$ \\
\hline Identificação do ônibus interno & 4 & $3 \%$ \\
\hline Ter um aplicativo de celular com os horários (site da UFSM) & 4 & $3 \%$ \\
\hline Subtotal & 45 & $33 \%$ \\
\hline \multicolumn{3}{|l|}{ Sobre o veículo } \\
\hline $\begin{array}{l}\text { Manutenção dos veículos (reforma interna, modernizar o ônibus, } \\
\text { higiene) }\end{array}$ & 20 & $15 \%$ \\
\hline $\begin{array}{l}\text { Maior cautela na condução do veículo (melhorar a segurança do } \\
\text { veículo) }\end{array}$ & 4 & $3 \%$ \\
\hline Sistema de climatização interna & 3 & $2 \%$ \\
\hline Sistema de campainha para solicitar parada & 3 & $2 \%$ \\
\hline $\begin{array}{l}\text { Instalação de puxadores ou barras para as pessoas em pé se } \\
\text { segurarem }\end{array}$ & 2 & $1 \%$ \\
\hline Promover acessibilidade ao veículo & 2 & $1 \%$ \\
\hline Subtotal & 34 & $25 \%$ \\
\hline Total & 208 & - \\
\hline
\end{tabular}

\section{CONCLUSÃO}

A pesquisa mostrou os principais pontos de satisfação e insatisfação dos usuários do transporte interno da UFSM, além de identificar o perfil dos usuários e a frequência de utilização deste serviço. O serviço precisa ser melhor informado aos 
usuários, pois $20 \%$ dos respondentes à pesquisa não conhecem o serviço de ônibus interno da UFSM. Os principais motivos de uso do serviço são economia de tempo, cursos e/ou disciplinas em locais diferentes, e o cansaço pelo deslocamento a pé. $\mathrm{O}$ predomínio de uso do serviço é o diurno, podendo supor que a divulgação junto ao turno noturno deve ser intensificada.

Quanto à satisfação dos usuários entre os itens pesquisados, os usuários mostraram maior insatisfação no item climatização interna do veículo e no estado de conservação dos ônibus, sendo, portanto, estes itens que devem prioritariamente serem melhorados. Já os demais itens: horário, frequência e rota de circulação do ônibus, quantidade de lugares e pontualidade; foram itens com um índice de satisfação alto, mas que devem ser mantidos e melhorados. Nas sugestões, observam-se pontos levantados que reforçam os diretamente interrogados. Há a confirmação de que a preocupação com o estado físico do veículo é um ponto a ser melhorado, além da maior divulgação do serviço e os ajustes na rota são necessários, abrangendo principalmente aspectos de percurso e horários/frequência.

A seguir estão relacionadas algumas ações levantadas no estudo recomendadas para melhorar a satisfação no serviço de ônibus interno da UFSM:

- Ampliar a divulgação do serviço, da rota e dos horários. Algumas sugestões: aplicativo de celular com dados e atualizações sobre o serviço; sinalização informativa das rotas e das paradas; identificação visual dos veículos;

- Melhorar a conservação do serviço, iniciando pela higienização, revisão mecânica, reforma interna e externa, adequação às necessidades do serviço, como campainha de parada, puxadores para segurança dos passageiros em pé, itens de segurança e conforto e climatização interna;

- Promover acessibilidade ao veículo para portadores de necessidades especiais;

- Divulgar o serviço oferecido (percurso, horário) para os estudantes de cursos noturnos;

- Estudar rotas alternativas e/ou alternadas;

- Estudar a demanda em horários de pico, com frequências de partida diferente;

- Adequação dos horários dos veículos em sintonia com os horários de aula, incluindo um horário as $7 \mathrm{~h}$ da manhã. 


\section{REFERÊNCIAS}

CASTRO A. Gerenciamento da mobilidade: uma contribuição metodológica para a definição de uma política integrada dos transportes para o Brasil. 2005.

DEMING WE. Qualidade: a revolução da administração. Rio de Janeiro: Marques Saraiva, 1990, $367 \mathrm{p}$.

ENGEL JF. et al. Comportamento do Consumidor. Rio de Janeiro: LTC, 2000.

JURAN JM. Juran na liderança pela qualidade. São Paulo: Pioneira, 1990, 386 p.

LIMA JUNIOR OF. Qualidade em serviços de transportes: conceituação e procedimentos para diagnóstico. São Paulo, Tese (Doutorado). Escola Politécnica, Universidade de São Paulo, 1995, 215 p.

MCDANIEL C, GATES R. Fundamentos de pesquisa de marketing. $4^{\text {a }}$ edition, Rio de Janeiro, 2005.

RAIA JUNIOR AA. Acessibilidade e mobilidade na estimativa de um índice de potencial de viagens utilizando redes neurais artificiais e sistemas de informação. Tese, Doutorado em Engenharia Civil/ Transportes pela Universidade de São Paulo, Escola de Engenharia de São Carlos, São Carlos, 2000.

PARRA CM. Gerenciamento da mobilidade em Campi Universitários: Problemas, Dificuldades e Possíveis Soluções no Caso Ilha de Fundão/ UFRJ, Tese de M.Sc., COPPE/UFRJ, Rio de Janeiro, 2006.

PAULLEY N. et al. The Demand for Public Transport: The Effect Of Fares, Quality Of Service, Income And Car Ownership. Transport Policy. 2006;13(4):296-306.

TOBIAS MSG. et al. Desafios E Soluções Para Mobilidade Em Campus Universitário: Um Estudo De Caso Na Ufpa/ Belém/ PA. 2013.

UFSM. Site institucional. Disponível em: https://portal.ufsm.br/indicadores/ind. Acesso em 01/09/2015.

UFSM. Transporte interno e intercampi gratuito na UFSM. Disponível em: http://coral.ufsm.br/proinfra/index.phptransporte-gratuito. Acesso em 09/09/2015. 